\title{
Restoration of miR-7 expression suppresses the growth of Lewis lung cancer cells by modulating epidermal growth factor receptor signaling
}

\author{
JINGRONG LI ${ }^{1}$, YIJIE ZHENG ${ }^{2,3}$, GENGYUN SUN ${ }^{4}$ and SHUDAO XIONG ${ }^{5}$ \\ ${ }^{1}$ Department of Emergency, The Second Hospital of Anhui Medical University, Hefei, Anhui, P.R. China; \\ ${ }^{2}$ Department of Medicine, College of Physicians and Surgeons, Columbia University, New York, NY, USA; \\ ${ }^{3}$ Department of Immunology and Key Laboratory of Molecular Medicine of Ministry of Education, \\ Shanghai Medical College, Fudan University, Shanghai; ${ }^{4}$ Department of Respiratory Medicine, \\ The First Affiliated Hospital of Anhui Medical University, Hefei; ${ }^{5}$ Department of Hematology/Oncology, \\ The Second Hospital of Anhui Medical University, Hefei, Anhui, P.R. China
}

Received June 14, 2014; Accepted September 9, 2014

DOI: $10.3892 / o r .2014 .3519$

\begin{abstract}
RNAs are an abundant class of short endogenous non-coding RNAs that function as important regulators of multiple target genes and participate in diverse biological roles in carcinogenesis. However, the role of miR-7 in lung cancer remains unclear and requires further elucidation. In the present study, we found a reduction of miR-7 expression in Lewis lung cancer (3LL) cells originating from mice by real-time RT-PCR. Restoration of miR-7 inhibited 3LL cell proliferation, induced cell apoptosis in vitro and reduced tumorigenicity in vivo. We further confirmed that miR-7 downregulated the expression of both epidermal growth factor receptor (EGFR) and murine leukemia viral oncogene homologue-1 (RAF-1) oncogenes by real-time PCR and western blot analysis. Furthermore, inhibition of EGFR showed similar effects to miR-7 enforcement in 3LL cells. Taken together, these findings revealed that miR-7 acts as an antitumor miRNA in 3LL by targeting and suppressing the expression of both EGFR and RAF-1 oncogenes. This study may provide a rationale for the use of miR-7 in lung cancer target therapy.
\end{abstract}

\section{Introduction}

microRNAs are an abundant class of short endogenous non-coding RNAs that act as important post-transcriptional

Correspondence to: Dr Gengyun Sun, Department of Respiratory Medicine, The First Affiliated Hospital of Anhui Medical University, 218 Jixi Road, Hefei, Anhui 230022, P.R. China

E-mail: sungengyun@tom.com

Dr Shudao Xiong, Department of Hematology/Oncology, The Second Hospital of Anhui Medical University, 678 Furong Road, Hefei, Anhui 230601, P.R. China

E-mail:xshdao@163.com

Key words: microRNA, miR-7, epidermal growth factor receptor, RAF-1, oncogene regulators of gene expression involved in crucial biological processes, including development, differentiation, proliferation and apoptosis. microRNAs inhibit their target gene expression through imperfect pairing with their target messenger of protein-coding genes (1-4). Growing evidence suggests that miRNAs are aberrantly expressed in many human cancers, which may act as oncogenes or tumor suppressors in human cancer including lung carcinoma (5-7).

miR-7 has been identified to be dysregulated in some human cancers and is functionally associated with cancer cell proliferation, differentiation and apoptosis $(8,9)$. Recent studies also demonstrated miR-7 to be a potential tumor suppressor in several human cancers including brain, colon and liver (8-10). However, the biological function of miR-7 in lung cancer and its molecular mechanism remain to be further elucidated. In addition, to the best of our knowledge, little information is available on the miR-7 expression profiles and function in the well-defined animal model specimens including Lewis lung cancer (3LL).

Therefore, in the present study, we described and characterized the expression and function of miR-7 in the $3 \mathrm{LL}$ cell line originating from mouse lung cancer compared with normal lung tissues. We showed that the expression of mature miR-7 is downregulated in 3LL cells compared to normal lung tissues. Restoration of its expression triggered a considerable decrease in cell proliferation and induced apoptosis in 3LL cells in vitro. This type of miR-7 restoration also significantly reduced tumorigenicity in vivo. With regard to its mechanism, miR-7 directly targeted the oncogene epidermal growth factor receptor (EGFR) and murine leukemia viral oncogene homologue-1 (RAF-1) and downregulated their expression. Collectively, our results suggest miR-7 to be a tumor suppressor gene that suppresses the growth of 3LL cells.

\section{Materials and methods}

Cell lines and cell culture. The 3LL cell line (murine Lewis lung cancer cell line) was provided by the Institute of Biochemistry 
and Cell Biology of the Chinese Academy of Science, China. $3 \mathrm{LL}$ cells were cultured at $37^{\circ} \mathrm{C}$ under $5 \% \mathrm{CO}_{2}$ in a complete RPMI-1640 (31800089) medium containing 10\% heat-inactivated fetal bovine serum (10100139) and supplemented with 2mM glutamine (21051040) (all from Gibco, Grand Island, NY, USA), $100 \mathrm{IU} / \mathrm{ml}$ penicillin and $100 \mu \mathrm{g} / \mathrm{ml}$ streptomycin sulfate (Shanghai No. 4 Pharmaceutical Factory, China).

RNA extraction. Total RNA of cultured cells and normal lung tissues from mice were extracted with TRIzol reagent (15596-026; Invitrogen, Carlsbad, CA, USA) according to the manufacturer's protocol. RNAs were quantitated and then stored at $-80^{\circ} \mathrm{C}$ prior to RT-PCR analysis.

Quantitative RT-PCR ( $q R T-P C R)$ for miRNA. For mature miRNA expression analysis, $\sim 10$ ng of total RNA was converted to cDNA using the PrimerScript RT reagent kit (DRR037A; Takara, Dalian, China) with miR-7-specific and U6 primers (RiboBio, Guangzhou, China). After reverse transcription, qRT-PCR was performed using FastStart Universal SYBR-Green Master kit (04913850001; Roche, Mannheim, Germany) with Bulge-Loop ${ }^{\mathrm{TM}}$ miR-7 qRT-PCR primers (RiboBio) on the ABI 7500 Thermocycler (Applied Biosystems, Foster City, CA, USA) according to the manufacturer's protocol. U6 gene was used as a normalization control for all samples.

miRNA mimics and transfection. The mature miR-7 duplex mimics (miR-7) and negative control oligonucleotide duplex mimics (miR-NC) were designed and provided by RiboBio. Confluent 3LL cells (30-50\%) were transfected with miRNAs by Lipofectamine 2000 (11668-019; Invitrogen) according to the manufacturer's protocol. Total RNA was extracted $24 \mathrm{~h}$ after transfection, and total cell proteins were extracted 48 or $72 \mathrm{~h}$ after transfection.

Cell proliferation assay. Cell proliferation assay was determined by Cell Counting Kit-8 assay (CK04-11; Dojindo, Japan), a redox assay similar to 3-(4,5-dimethylthiazol2-yl)-2,5-diphenyltetrazolium bromide (MTT) according to the manufacturer's protocol. Cell proliferation assay was carried out in hexakis.

Apoptotic morphology by DAPI staining. Cells were stained with 4,6-diamidino-2-phenylindole (DAPI) (40728ES10; Yeasen, Shanghai, China) and cells with fragmented or condensed nuclei were defined as apoptotic cells. At least three visual fields were observed under a fluorescence microscope for each sample.

$q R T-P C R$ for $m R N A$ expression. Synthesis of cDNA was performed on $1 \mu \mathrm{g}$ of total RNA per sample with the PrimerScript RT reagent kit according to the manufacturer's manual. qRT-PCR was performed in triplicate for each sample by FastStart Universal SYBR-Green Master kit (04913850001; Roche) according to the manufacturer's instructions. Oligonucleotides were designed by the PrimerExpress software 2.0 and synthesized by Sangon Biotech (Shanghai, China). GAPDH was used as a housekeeping gene for normalization. The sequences of primers in this section were: (EGFR forward, 5'-CCAACTATGGGACAAACAGAA-3' and reverse,

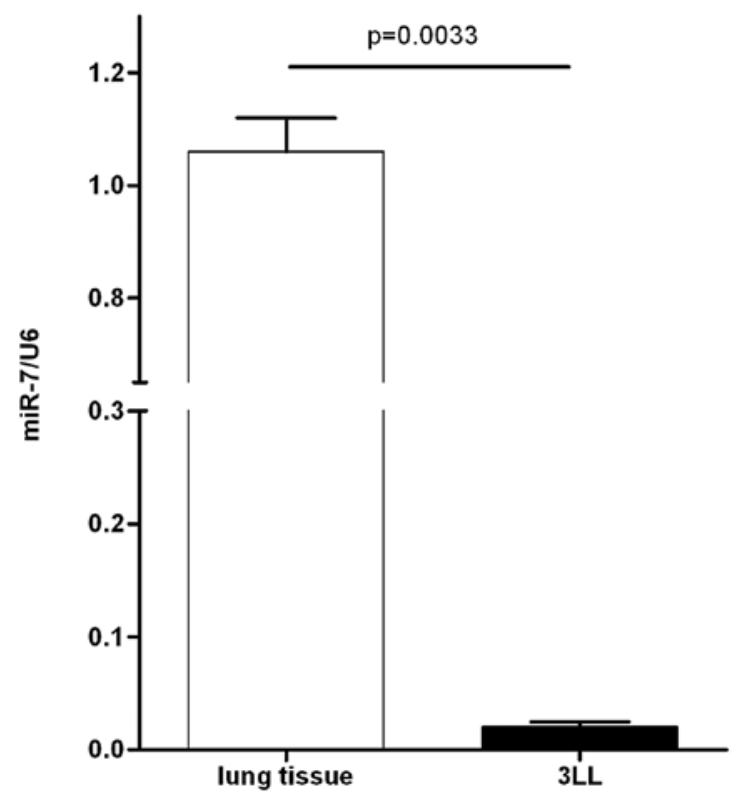

Figure 1. miR-7 is downregulated in Lewis lung cancer (3LL) cells. miR-7 expression was detected by real-time PCR in 3LL cells and mouse normal lung tissue. Each sample was analyzed in triplicate.

5'-ATCGCACAGCACCAATCA-3'; RAF-1 forward, 5'-AGTCACGCTGGAGTGGTTCT-3' and reverse, 5'-GTCTCGGTTGTTGATGTGGG-3'; GAPDH forward, 5'-TGCACCACCAACTGCTTAGC-3' and reverse, 5'-GCA TGGACTGTGGTCATGAG-3'.

Western blot analysis. Proteins extracted from cells were immunoblotted with different antibodies following a published protocol $(11,12)$. The primary antibodies used were EGFR and RAF-1 (1:3,000 dilutions), GAPDH (1:10,000 dilutions) (Cell Signaling, Danvers, MA, USA).

Tumorigenicity assay in mice. C57/BL/6 mice (4-6 weeks old) were purchased from Shanghai Experimental Center, Chinese Academy of Science. All animal experiments were carried out in compliance with the Guide for the Care and Use of Laboratory Animals published by the US National Institutes of Health (NIH publication no. 85-23, revised 1996) and the Guidelines of Anhui Medical Laboratory Animal Care and Use Committee. miR-7- and miR-NC-transfected $3 \mathrm{LL}$ tumor cells $\left(5 \times 10^{4}\right)$ per mouse were injected subcutaneously (s.c.) into either side of the posterior flank of the same C57/BL/6 female mouse. After 7 days, the tumor volume was measured with a vernier caliper at weekly intervals. The tumor volume $=\mathrm{W}^{2} \mathrm{xL} / 2$, where $\mathrm{W}$ is the short diameter and $\mathrm{L}$ is the long diameter.

Statistical analysis. Data are expressed as the means + SEM of three independent experiments. For all statistical tests, PRISM 5.0 (GraphPad Software Inc., San Diego, CA, USA) was used. miR-7 data between two groups was calculated using t-test analysis. ANOVA analysis was used for the multigroup comparison while the Student's t-test was applied for the comparison between two groups. P-values $<0.05$ were considered to indicate statistically significant differences. 
A

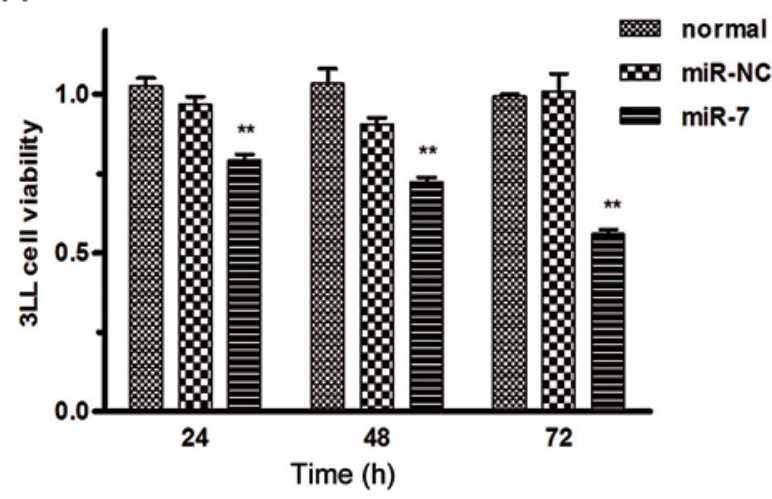

B

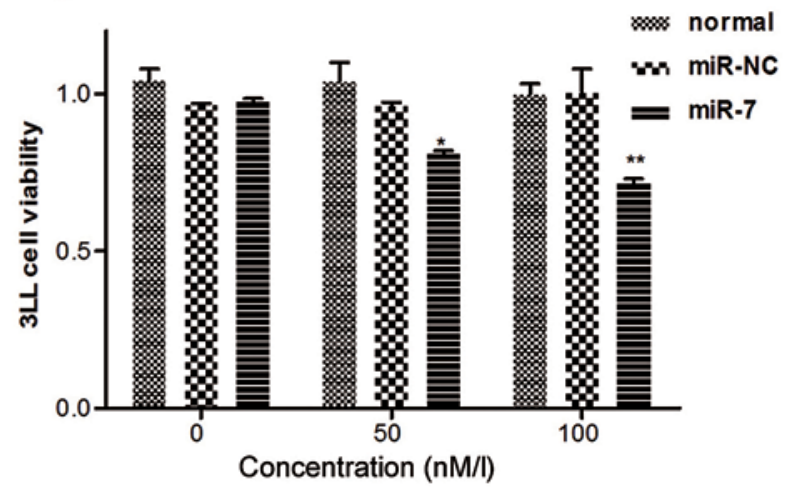

C

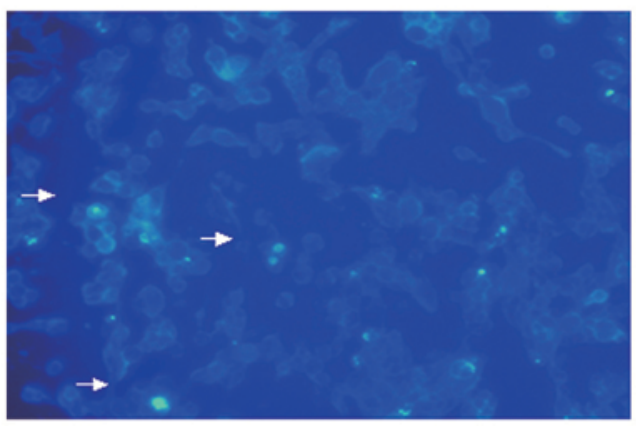

miR-NC
D

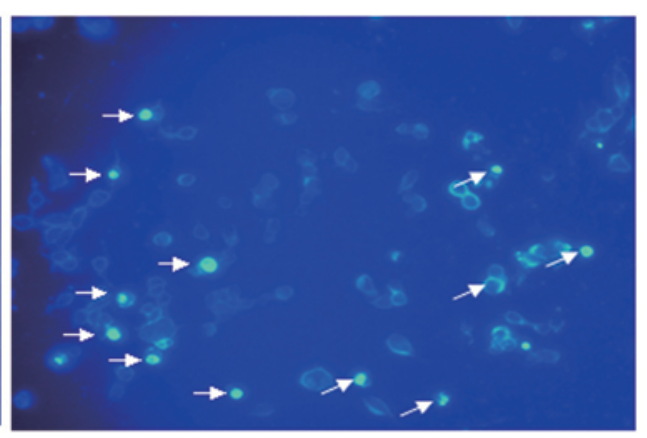

miR-7
E

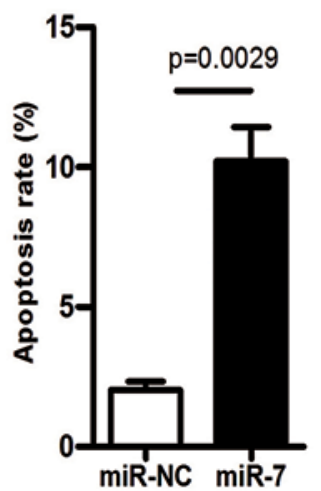

Figure 2. miR-7 inhibits Lewis lung cancer (3LL) cell growth and induces cell apoptosis. (A) $3 \mathrm{LL}$ cells transfected with 50 nM miR-7 or negative control mimics (miR-NC) for 24, 48 and $72 \mathrm{~h}$; cell viability was evaluated by CCK-8 assay. (B) $3 \mathrm{LL}$ cells transfected with different doses ( 0,50 or $100 \mathrm{nM}$ ) of miR-7 mimics for $48 \mathrm{~h}$; cell viability was evaluated by CCK-8 assay. 3LL cells were transfected with $50 \mathrm{nM}$ of (D) miR-7 or (C) miR-NC, cells were incubated with low-serum (3\%) RPMI-1640 medium for $48 \mathrm{~h}$. The morphology of apoptotic cell nuclei was observed by DAPI staining using a fluorescence microscope. The 3LL cell (arrow) shows the morphology of the apoptotic cell nuclei. (E) Apoptotic rate in DAPI staining assay was analyzed. Data are representative of three independent experiments $\left({ }^{*} \mathrm{p}<0.05\right.$ and $\left.{ }^{* *} \mathrm{p}<0.01\right)$.

\section{Results}

miR-7 is significantly downregulated in $3 L L$ cells. To assess the biological role of miR-7 in the 3LL model, we first examined the expression of miR-7 by quantitative real-time PCR in 3LL cells and mouse lung normal tissues. As a result, miR-7 showed significantly lower expression in 3LL cells than in normal lung tissues (Fig. 1), indicating that the downregulation of miR-7 may be involved in mouse 3LL carcinogenesis.

Restoration of miR-7 decreases the proliferation and induces apoptosis of $3 L L$ cells in vitro. Since miR-7 was significantly downregulated in 3LL cells compared with normal lung tissues, miR-7 may serve as a tumor suppressor. Therefore, we next investigated the effect of miR-7 on phenotypes of $3 \mathrm{LL}$ cells. 3LL cells were transiently transfected with mature miR-7 mimic (miR-7). Control cells were also transfected with negative control mimic (miR-NC) without specifically targeting any mouse gene products. After $48 \mathrm{~h}$, cells transfected with miR-7 grew more slowly than the negative control and normal group in CCK-8 proliferation assay. 3LL cells also exhibited reduced cell proliferation in the presence of miR-7 in a time(Fig. 2A) and dose-dependent manner (Fig. 2B).
To further explore whether the reduced cancer cell number was due to cell apoptosis, we performed DAPI staining assay. 3LL cells were transfected with miR-7 mimic $(50 \mathrm{nM})$ or negative control mimic (50 $\mathrm{nM}$ ) in low-serum (3\%) RPMI-1640 medium for $48 \mathrm{~h}$ and subsequently analyzed by DAPI staining assay. We observed considerably more apoptotic cells in miR-7-transfected 3LL cells (Fig. 2D) than in negative control cells (Fig. 2C). The percentage of cells with apoptotic nuclei significantly increased in miR-7-transfected 3LL cells compared to the control cells in DAPI staining (Fig. 2E). Taken together, these results indicate that the inhibition of cell proliferation by miR-7 is associated with the increased 3LL cell apoptosis.

miR-7 inhibits tumor growth of $3 L L$ cells in vivo. Considering the inhibition of 3LL cell proliferation by miR-7 in vitro, we further evaluated the effect of miR-7 on tumor growth of 3LL cells in vivo. 3LL cells were transfected either with miR-7 or miR-NC. After $6 \mathrm{~h}$, they were implanted subcutaneously into either posterior flank of the same C57BL/6J mice ( $5 \times 10^{4}$ cells/injection site). 3LL cells transfected with miR-NC formed tumors 7 days after implantation. However, 3LL cells transfected with miR-7 partially failed to grow 7 days after injection. Furthermore, cells transfected with miR-7 exhibited 
A

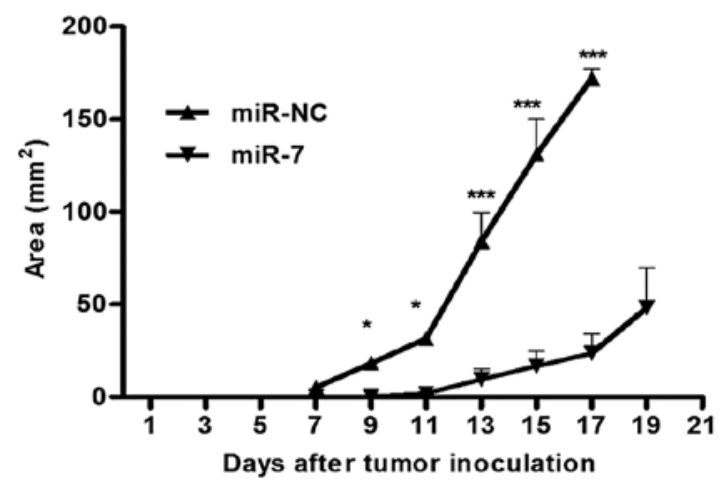

B

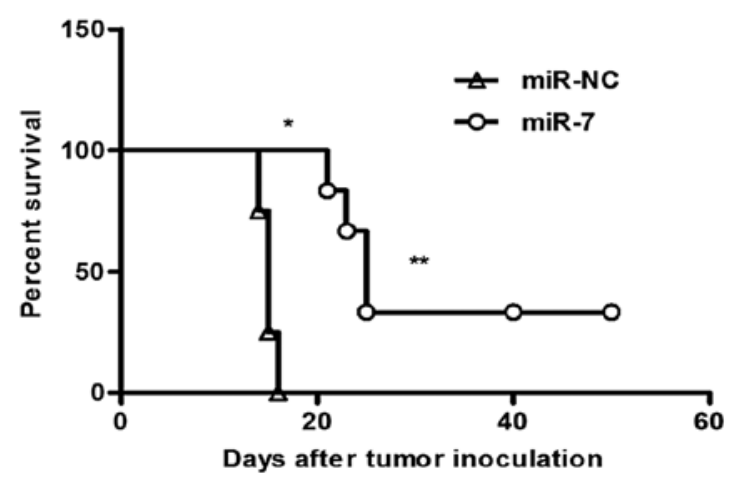

Figure 3. miR-7 inhibits 3LL Lewis lung tumor growth in vivo. miR-7- and miR-NC-mimic-transfected 3LL cells (5x10 $\left.{ }^{4}\right)$ were injected subcutaneously (s.c.) into either side of the posterior flank of the same female C57/BL/6 mouse respectively. (A) Tumor sizes are shown as mean + SEM. (B) The curve of survival rates was recorded. Three mice were used at each time and repeated twice, for a total of six mice. Data are representative of two independent experiments $\left(\right.$ p $<0.05,{ }^{* *} \mathrm{p}<0.01$ and $\left.{ }^{* * *} \mathrm{p}<0.001\right)$.

A

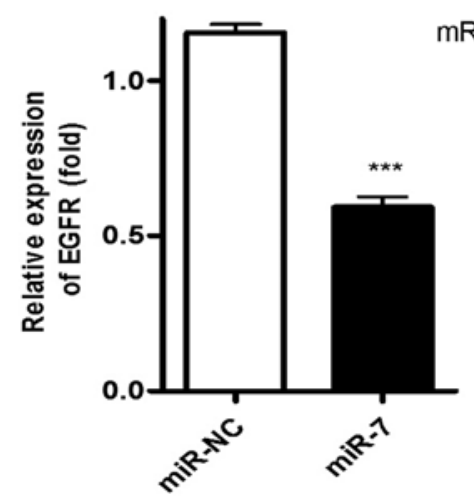

B

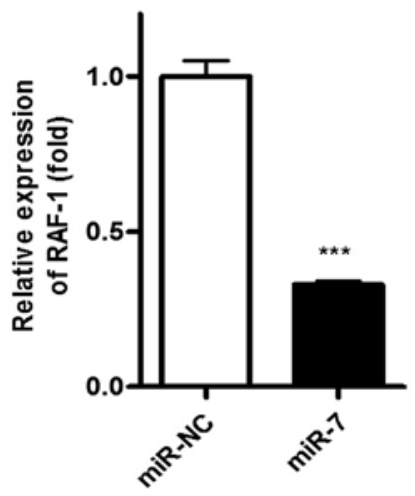

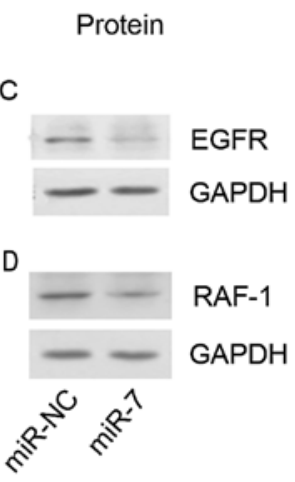

Figure 4. miR-7 suppresses the expression of EGFR and RAF-1 in Lewis lung cancer (3LL) cells. 3LL cells were transfected with miR-7 or miR-NC at $50 \mathrm{nM}$ for 24 or $72 \mathrm{~h}$. (A and B) mRNA expression levels of EGFR and RAF-1 were measured by real-time RT-PCR and standardized to GAPDH. (C and D) Protein levels of EGFR and RAF-1 were determined by western blot with GAPDH used as a loading control (one of three similar blots is shown) $(* * * * 0.001)$.

a marked reduction in tumor size (Fig. 3A) and an increase in survival rate (Fig. 3B) compared to the control group. These data indicate that elevated miR-7 level in 3LL cells markedly reduce their ability to form tumors.

miR-7 suppresses its target gene expression of EGFR and $R A F-1$ in $3 L L$ cells. We next investigated the molecular mechanism through which miR-7 inhibits cancer cell proliferation and induces cell apoptosis. Using bioinformatics analysis, we found the potential biding sites of EGFR and RAF-1 (data not shown). To further assess whether miR-7 had a functional role in regulating these two targets (EGFR and RAF-1) in 3LL, 3LL cells were transfected with miR-7 mimic for 24 or $72 \mathrm{~h}$ and we then analyzed the EGFR and RAF-1 expression by real-time RT-PCR and western blot analysis. As a result, overexpression of miR-7 markedly reduced the expression of EGFR and RAF-1 at mRNA (Fig. 4A and B) and protein level (Fig. 4C and D) compared with the control groups. Taken together, these results suggest that miR-7 decreases expression of EGFR and RAF-1 through its direct negative regulation in 3LL cells originating from mice.
Inhibition of EGFR suppresses the proliferation and induces apoptosis of $3 L L$ cells in vitro. The above data showed that miR-7 downregulated the expression of EGFR and its downstream RAF-1. We next examined whether such type of inhibition is involved in the effect of miR-7 on 3LL cells. As shown in Fig. 5 blocking EGFR with its inhibitor AST-1306 (Selleck, USA) (3 nM) significantly decreased the cell viability of $3 \mathrm{LL}$ cells in CCK-8 assay (Fig. 5A), and also induced 3LL cell apoptosis in DAPI staining assay (Fig. 5B). These results were consistent with the effect of miR-7 on 3LL cells (Fig. 2A, B and D), providing further evidence that miR-7 mediates cell proliferation suppression and induces apoptosis by downregulation of EGFR in 3LL cells.

\section{Discussion}

Increasing evidence suggests that miRNAs play a key regulatory role in cancer-related biological effectors $(13,14)$, and their abnormal expression is closely related to various cancers, including lung cancer $(1,15,16)$. miRNAs are directly involved in cancer initiation and progression by directly regulating 
A

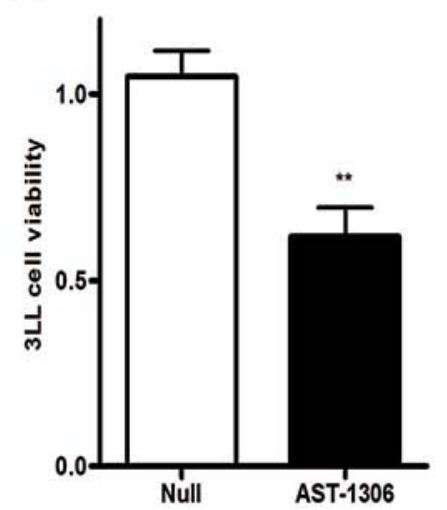

B

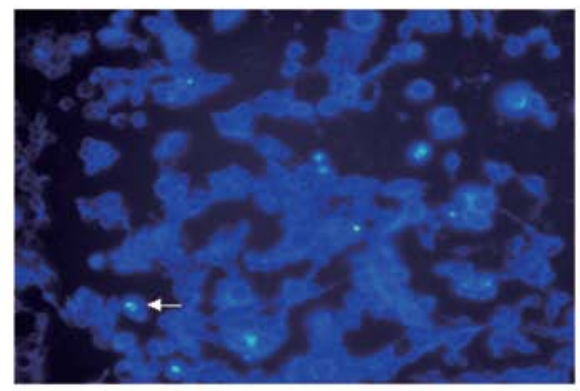

Null
C

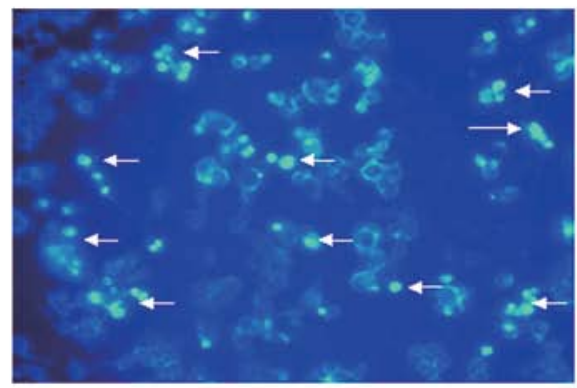

AST-1306

Figure 5. EGFR inhibitor AST-1306 induces apoptosis of Lewis lung cancer (3LL) cells. 3LL cells were treated with EGFR inhibitor AST-1306 (3 nM) or DMSO (Null) for 24 h. (A) Cell viability was detected by CCK-8 assay. (B and C) Apoptotic nuclei were observed and analyzed by DAPI staining. The 3LL cell (arrow) shows the morphology of the apoptotic cell nuclei. Data are representative of three independent experiments $\left({ }^{* *} \mathrm{p}<0.01\right)$.

the expression of important cancer-related genes, thereby functioning as tumor suppressors or oncogenes $(17,18)$. For example, miR-21, which is highly expressed in pancreatic and breast cancer, promotes tumor cell growth by inhibiting the tumor suppressor PTEN $(19,20)$. On the contrary, miR-7, which has low expression in human brain, liver, breast and lung cancer, often acts as a tumor suppressor (9,21-23). To date, however, in a mouse cancer model, the expression and function of miR-7 has not been fully elucidated. In this study, the expression of miR-7 in 3LL cells from murine Lewis lung cancer was significantly reduced. 3LL cell proliferation was markedly suppressed and cell apoptosis was also induced in vitro following administration of miR-7. Markedly, miR-7 could also decrease the tumorigenicity in C57BL/6J in vivo. Therefore, our data suggested miR-7 to be a tumor suppressor in 3LL cell lines, which is consistent with that of the human A549 cell lines in our previous study $(23,24)$. However, another study also showed that miR-7 promoted, rather than inhibited, cell growth and tumor formation in human lung cancer line CL1-5 (25). Therefore, the role of miR-7 may vary in different cancer types according to the biological background of the tumor cells themselves and the main target genes involved in this process $(13,14)$.

Using bioinformatics analysis, we also found the potential target genes including EGFR and RAF-1 in murine species due to the high conservatism of microRNA. EGFR is a transmembrane glycoprotein that plays important roles in cancer growth, including non-small cell lung cancer (NSCLC). Its ligands, such as epidermal growth factor (EGF) or transforming growth factor- $\alpha$ (TGF- $\alpha$ ), cause EGFR to undergo a conformational change leading to autophosphorylation of EGFR and activation of the EGFR growth factor pathway (26). The elevated expression and activity of EGFR in cancer increase cell proliferation and angiogenesis and inhibit programmed cell death. EGFR is expressed in $40-80 \%$ of NSCLC and is associated with development, progression and treatment resistance (27). Previous studies demonstrated that miR-7 downregulates EGFR expression in a range of cancer cell types via its specific interaction with the EGFR mRNA 3'-untranslated region (3'-UTR) (21). These results are further supported by the present finding that miR-7 downregulated EGFR expression and reduced cell growth in vitro and in vivo $(28,29)$. Notably, a previous study indicated that EGFR could also upregulate miR-7 expression (28). Therefore, the interaction of miR-7 and EGFR appears as a feedback balance, and the outcome of the balance among the target gene network determine the initiation and development of tumor.

The serine/threonine kinase RAF-1 is a downstream effecter of EGFR signaling. It is commonly activated by mutations and is involved in the regulation of tumor cell proliferation, survival and migration. RAF-1 is overexpressed in human cancers including lung carcinoma, therefore it is emerging as a promising target for cancer therapy (30-33). In the present study, we showed that the potential target RAF-1 is significantly downregulated by enforcement of miR-7 expressing in 3LL cell lines. These results are similar to a study by Webster et al (33). Thus, miR-7 decreases the expression of both EGFR and its downstream target RAF-1 and then inhibits 3LL cell growth. Furthermore, blocking EGFR exhibited similar effects with miR-7 in 3LL cells. Therefore, identification of both EGFR and its downstream target RAF-1 as the miR-7 target gene maybe explain, at least in part, the molecular mechanism of the tumor suppressor miR-7 in murine 3LL.

In conclusion, through analysis of the expression and function of miR-7 in a mouse cancer model, we found for the first time that miR-7 has a tumor-suppressive function through inhibition of cell proliferation and induction of apoptosis in mouse 3LL cell lines. We also identified both EGFR and its downstream target RAF-1 as two targets possibly involved in miR-7-mediated growth suppression and apoptosis induction of 3LL cells. These findings may provide a basic rationale for the use of miR-7 in the treatment of lung caner.

\section{Acknowledgements}

The authors thank Dr Ronghua Liu (Department of Immunology, Fudan University, Shanghai, China) for their technical help and scientific discussions. This study was supported by the National Science Foundation of China (81272259). 


\section{References}

1. van Kouwenhove M, Kedde M and Agami R: MicroRNA regulation by RNA-binding proteins and its implications for cancer. Nat Rev Cancer 11: 644-656, 2011.

2. Ryan BM, Robles AI and Harris CC: Genetic variation in microRNA networks: the implications for cancer research. Nat Rev Cancer 10: 389-402, 2010.

3. Aguda BD, Kim Y,Piper-Hunter MG, Friedman A and Marsh CB: MicroRNA regulation of a cancer network: consequences of the feedback loops involving miR-17-92, E2F, and Myc. Proc Natl Acad Sci USA 105: 19678-19683, 2008.

4. Xu TP, Zhu CH, Zhang J, et al: MicroRNA-155 expression has prognostic value in patients with non-small cell lung cancer and digestive system carcinomas. Asian Pac J Cancer Prev 14: 7085-7090, 2013.

5. Yang S, Li Y, Gao J, et al: MicroRNA-34 suppresses breast cancer invasion and metastasis by directly targeting Fra-1. Oncogene 32: 4294-4303, 2013.

6. Hulf T, Sibbritt T, Wiklund ED, et al: Epigenetic-induced repression of microRNA-205 is associated with MED1 activation and a poorer prognosis in localized prostate cancer. Oncogene 32: 2891-2899, 2013.

7. Zhang Y, Chen X, Lian H, et al: MicroRNA-503 acts as a tumor suppressor in glioblastoma for multiple antitumor effects by targeting IGF-1R. Oncol Rep 31: 1445-1452, 2014.

8. Zhang N, Li X, Wu CW, et al: microRNA-7 is a novel inhibitor of YY1 contributing to colorectal tumorigenesis. Oncogene 32: 5078-5088, 2013.

9. Fang Y, Xue JL, Shen Q, Chen J and Tian L: MicroRNA-7 inhibits tumor growth and metastasis by targeting the phosphoinositide 3-kinase/Akt pathway in hepatocellular carcinoma. Hepatology 55: 1852-1862, 2012.

10. Saydam O, Senol O, Würdinger T, et al: miRNA-7 attenuation in Schwannoma tumors stimulates growth by upregulating three oncogenic signaling pathways. Cancer Res 71: 852-861, 2011

11. Xiong SD, Yu K, Liu XH, et al: Ribosome-inactivating proteins isolated from dietary bitter melon induce apoptosis and inhibit histone deacetylase-1 selectively in premalignant and malignant prostate cancer cells. Int J Cancer 125: 774-782, 2009.

12. Zheng Y, Xiong S, Jiang P, et al: Glucocorticoids inhibit lipopolysaccharide-mediated inflammatory response by downregulating microRNA-155: a novel anti-inflammation mechanism. Free Radic Biol Med 52: 1307-1317, 2012.

13. Qiu ZX, Wang L, Han J, et al: Prognostic impact of Raf-1 and p-Raf-1 expressions for poor survival rate in non-small cell lung cancer. Cancer Sci 103: 1774-1779, 2012.

14. Mukherjee R, Bartlett JM, Krishna NS, Underwood MA and Edwards J: Raf-1 expression may influence progression to androgen insensitive prostate cancer. Prostate 64: 101-107, 2005.

15. Aurora AB, Mahmoud AI, Luo X, et al: MicroRNA-214 protects the mouse heart from ischemic injury by controlling $\mathrm{Ca}^{2+}$ overload and cell death. J Clin Invest 122: 1222-1232, 2012.

16. Croce CM: Causes and consequences of microRNA dysregulation in cancer. Nat Rev Genet 10: 704-714, 2009.

17. Khoshnaw SM, Green AR, Powe DG and Ellis IO: MicroRNA involvement in the pathogenesis and management of breast cancer. J Clin Pathol 62: 422-428, 2009.
18. Andorfer CA, Necela BM, Thompson EA and Perez EA: MicroRNA signatures: clinical biomarkers for the diagnosis and treatment of breast cancer. Trends Mol Med 17: 313-319, 2011.

19. Gong C, Yao Y, Wang Y, et al: Up-regulation of miR-21 mediates resistance to trastuzumab therapy for breast cancer. J Biol Chem 286: 19127-19137, 2011.

20. Chan LW, Wang FF and Cho WC: Genomic sequence analysis of EGFR regulation by microRNAs in lung cancer. Curr Top Med Chem 12: 920-926, 2012.

21. Kefas B, Godlewski J, Comeau L, et al: microRNA-7 inhibits the epidermal growth factor receptor and the Akt pathway and is down-regulated in glioblastoma. Cancer Res 68: 3566-3572, 2008.

22. Reddy SD, Ohshiro K, Rayala SK and Kumar R: MicroRNA-7, a homeobox D10 target, inhibits p21-activated kinase 1 and regulates its functions. Cancer Res 68: 8195-8200, 2008.

23. Xiong S, Zheng Y, Jiang P, et al: PA28gamma emerges as a novel functional target of tumour suppressor microRNA-7 in nonsmall-cell lung cancer. Br J Cancer 110: 353-362, 2014.

24. Xiong S, Zheng Y, Jiang P, Liu R, Liu X and Chu Y: MicroRNA-7 inhibits the growth of human non-small cell lung cancer A549 cells through targeting BCL-2. Int J Biol Sci 7: 805-814, 2011.

25. Chou YT, Lin HH, Lien YC, et al: EGFR promotes lung tumorigenesis by activating miR-7 through a Ras/ERK/Myc pathway that targets the Ets2 transcriptional repressor ERF. Cancer Res 70: 8822-8831, 2010.

26. Navolanic PM, Steelman LS and McCubrey JA: EGFR family signaling and its association with breast cancer development and resistance to chemotherapy (Review). Int J Oncol 22: 237-252, 2003.

27. Sonnweber B, Dlaska M, Skvortsov S, Dirnhofer S, Schmid T and Hilbe W: High predictive value of epidermal growth factor receptor phosphorylation but not of EGFRvIII mutation in resected stage I non-small cell lung cancer (NSCLC). J Clin Pathol 59: 255-259, 2006.

28. Mukohara T, Kudoh S, Yamauchi S, et al: Expression of epidermal growth factor receptor (EGFR) and downstreamactivated peptides in surgically excised non-small-cell lung cancer (NSCLC). Lung Cancer 41: 123-130, 2003.

29. Bozzetti C, Tiseo M, Lagrasta C, et al: Comparison between epidermal growth factor receptor (EGFR) gene expression in primary non-small cell lung cancer (NSCLC) and in fine-needle aspirates from distant metastatic sites. J Thorac Oncol 3: 18-22, 2008.

30. Lee KM, Choi EJ and Kim IA: microRNA-7 increases radiosensitivity of human cancer cells with activated EGFR-associated signaling. Radiother Oncol 101: 171-176, 2011.

31. Kalinowski FC, Giles KM, Candy PA, et al: Regulation of epidermal growth factor receptor signaling and erlotinib sensitivity in head and neck cancer cells by miR-7. PLoS One 7: e47067, 2012.

32. Duex JE, Comeau L, Sorkin A, Purow B and Kefas B: Usp18 regulates epidermal growth factor (EGF) receptor expression and cancer cell survival via microRNA-7. J Biol Chem 286: 25377-25386, 2011.

33. Webster RJ, Giles KM, Price KJ, Zhang PM, Mattick JS and Leedman PJ: Regulation of epidermal growth factor receptor signaling in human cancer cells by microRNA-7. J Biol Chem 284: 5731-5741, 2009. 